\title{
CONSTITUCIÓN Y MERCADO EN LA CRISIS DE LA INTEGRACIÓN EUROPEA: CONSIDERACIONES PRELIMINARES
}

\author{
José Luis García Guerrero y María Luz Martínez Alarcón ${ }^{1}$
}

doi: 10.18543/ed-64(1)-2016pp15-21

La primera aproximación de este investigador al fenómeno de las integraciones económicas se produjo en el año $2005^{2}$, por encargo del Senado de la República Dominicana en el marco de un Congreso Internacional que debía servir para impulsar la aprobación de una nueva Constitución en aquel país que finalmente vería la luz en el año 2010.

Desde un principio resultó evidente que el estudio que las integraciones económicas debía enmarcarse en el contexto de una globalización, perseguida intensamente tras la caída del muro de Berlín. La liberación multilateral del comercio promovida por la Organización Mundial del Comercio había fracasado y se buscaba alcanzar ese mismo objetivo profundizando en las integraciones económicas regionales supra estatales.

En aquella primigenia fase de estudio ya se pudo concluir, siguiendo a Weber y García Pelayo ${ }^{3}$, que la constitución racional normativa, también

${ }^{1}$ José Luis García Guerrero (IP1) y María Luz Martínez Alarcón (IP2) son profesores titulares de Derecho constitucional de la Facultad de Derecho de Albacete (Universidad de Castilla-La Mancha) e Investigadores Principales del Proyecto de Investigación Constitución y mercado en la crisis de la integración europea. $\mathrm{I}+\mathrm{D}+\mathrm{i}$ del Programa Estatal de Investigación, Desarrollo e Innovación orientada a los Retos de la Sociedad, del Ministerio de Economía y Competitividad (DER2013-48327-C3-1-R).

2 García Guerrero, J. L., «Integración económica y reforma constitucional», en el libro colectivo Visión y análisis comparativo de las reformas constitucionales en Iberoamérica, ONU, PUND, Santo Domingo 2005, pp. 135-155.

3 WeBER, M., Historia económica general, México 1942, p. 297, entre otras; GARCía PELAYo, Manuel, Derecho Constitucional Comparado, Alianza Editorial, Madrid, 1984, p. 38. 
denominada liberal, estaba al servicio del sistema económico capitalista. Se diferenciaban tres modelos de constitución económica: la liberal, la socialista y la intervencionista europea. Se partía de cuatro fases de integración: Acuerdo de libre comercio, Unión aduanera, Mercado común y Unidad económica supra estatal. Su estudio y diferenciación se realizaba desde una perspectiva constitucional y permitía obtener dos reglas y una conclusión. La primera regla demostraba que a medida que se intensificaba la integración importantes elementos básicos del concepto liberal de constitución resultaban progresivamente afectados. La segunda regla mostraba que la sintonía ideológica de las constituciones económicas de los Estados integrados atenuaba las afecciones al modelo constitucional, lo que se manifestaba de forma especialmente evidente en el caso de la Unión Europea. La conclusión permitía concretar cómo resultaban menoscabada la soberanía popular, la distinción entre poder constituyente y constituido, así como el principio democrático a medida que se profundizaba en las fases de integración y cómo esta afección se agravaba cuando faltaba la sintonía ideológica entre las constituciones económicas de los Estados integrados.

Una buena parte de la doctrina constitucional española, en los primeros años de esta década, mostraba su creciente preocupación por las consecuencias de la precipitada reforma del artículo 135 de la Constitución, así como, en muy directa relación, por el ataque a los derechos sociales y a los de los trabajadores. Para la doctrina española, estos problemas provenían de la Unión Europea y de sus concretas políticas económicas para hacer frente, probablemente, a la mayor crisis económica que vivía Europa desde la Segunda Guerra Mundial (no hay duda respecto a los periféricos). La afección a los derechos de los trabajadores, al Estado del bienestar y la regla constitucional de la estabilidad presupuestaria centraron la mayor parte de los estudios (sirva de muestra el realizado por la IP 2 de este proyecto de investigación junto a Diego López Garrido ${ }^{4}$, a propósito de esta última materia).

En este contexto se realiza, al inicio de 2013, la segunda aproximación a la materia que ya llevaba el revelador título de «Las integraciones económicas supra estatales y los acuerdos entre bloque económicos, en definitiva, la

${ }^{4}$ AA.VV., Reforma constitucional y estabilidad presupuestaria. El artículo 135 de la Constitución española, dirigido por Diego López Garrido y coordinado por María Luz Martínez Alarcón, CEPC, Madrid 2013. Asimismo, la IP 2 de este proyecto publicó, en este mismo año, el trabajo «La estabilidad presupuestaria en el marco de la globalización: algunas reflexiones sobre su compatibilidad con la ideología jurídica del welfare state», en el libro Constitución y Globalización. Transformaciones del Estado constitucional, coordinado por Fernando Reviriego Picón, Fundación Manuel Giménez Abad, Zaragoza 2013, pp. 165-200. 
globalización ${ }^{5}$ como último embate al concepto racional normativo de constitución $»^{6}$. Este texto, que es más bien una hipótesis de trabajo o ensayo y que ya anunciaba la necesidad de un enfoque multidisciplinar, sirvió de inspiración y guía al proyecto de investigación. Lo novedoso de la hipótesis de trabajo es tratar de poner de relieve que las afecciones al concepto racional normativo no se limitan a ciertas partes de las constituciones de los Estados miembros sino que inciden en la práctica totalidad de la Norma Fundamental. Además, no es la integración europea la única responsable, sino la globalización en su conjunto, que condiciona y determina la Constitución de un Estado miembro (asimétricamente) y la propia estructura constitucional de una integración. La Unión Europea, pese a su fortaleza (comparada con los Estados que la integran), tiene unos márgenes de discrecionalidad en la toma de sus decisiones fuertemente condicionados por la globalización. En ésta, ya desde 2013, eran discernibles dos etapas, una primera de integraciones regionales, que ha conocido su culminación con la Unión Europea, y una segunda fase reconocible por los acuerdos de libre comercio que firman las propias integraciones entre sí o éstas con potencias económicas, ex potencias, emergentes o simples Estados.

Con estos parámetros se afronta el proyecto de investigación «Constitución y mercado en la crisis de la integración europea», cuyo título, bajo los presupuestos delineados, no puede inducir a equívoco. No se trata de un problema europeo, sino mundial. En consecuencia, no puede afrontarse sólo en lo referente al concierto europeo, lo que es, sin duda, imprescindible, sino que debe abordarse en un contexto mundial, el de la globalización en sus dos fases. Además, la amplitud de la materia objeto de estudio ha exigido una integración de diversos equipos de investigación, tres de estos corresponden a la Universidad de Castilla Mancha, dos de constitucional y uno de economía aplicada que actúan como proyecto coordinador. Como proyectos coordinados aparece el de la Universidad de Deusto, que se centra, además, en la mutación de la constitución económica europea; y, finalmente, el de Universidad Pablo de Olavide que se ocupa, asimismo, de la afección de los derechos sociales en el ámbito autonómico. Completan el proyecto de investigación diversos investigadores nacionales, así como europeos y americanos,

${ }^{5}$ Con posterioridad a la publicación de este trabajo y a través del profesor Rubio Llorente, se conoció un trabajo de consulta obligatoria en la materia, Rodrik, Dani, La paradoja de la globalización, Antonio Bosch, Barcelona, 2011.

${ }^{6}$ Publicado en el libro, Constitución, Justicia constitucional y Derecho procesal constitucional (Liber Amicorum en Homenaje al profesor Dr. Juan Manuel Pellerano Gómez), coordinadores Jorge Prats, Eduardo y VALERIo Jimenián, Manuel, Instituto Dominicano de Derecho Constitucional y Librería Jurídica Internacional, Santo Domingo, República Dominicana, 2014, pp. 544-591. 
estos últimos integrados en el equipo de trabajo por prescripción ministerial. Actualmente trabajan en este proyecto de investigación más de sesenta investigadores de dieciséis universidades y no dejan de incorporarse relevantes especialistas en las distintas materias.

Este proyecto de investigación persigue un doble objetivo: establecer desde una perspectiva constitucional las afecciones que las dos etapas de la globalización originan al concepto racional normativo de Constitución y analizar qué medidas pueden adoptarse para subsanar o disminuir los efectos de estos embates. La integración europea ocupa un papel central en la investigación por ser la única que ha alcanzado plenamente la última fase: la Unidad económica y monetaria supraestatal. Su experiencia permite, con las cautelas y precauciones necesarias para evitar adoptar dominantemente el método fenomenológico, extrapolar problemas y soluciones a otros procesos de integración. Este rol central europeo se justifica asimismo por la búsqueda de soluciones, lo que nos conecta con el segundo objetivo final de la investigación. Los embates asimétricos que han sufrido las constituciones de los Estados miembros de la Unión Europea se presupone que dominantemente sólo pueden solucionarse mediante intensas reformas de su gobernanza, lo que requiere de las consecuentes propuestas de lege ferenda. Ahora bien, hay otra hipótesis posible que requiere también de exploración: que no sea posible subsanar los embates al concepto racional normativo de constitución mediante reformas y que sea preciso subsanar los problemas acudiendo a la construcción de un Estado Federal. Aunque, hay que precisar, pues se está lejos de construcciones irrealizables, la estructura federal debe presentar unos contenidos mínimos y determinados con los que superar los ataques que las constituciones liberales de los Estados miembros sufren. A los autores de estas líneas no se les escapan las dificultades y extensión de trabajo; sin entrar en profundidades, todos somos conscientes de las asimetrías de las afecciones a las constituciones de los Estados miembros y la actual ausencia de un demos europeo, entre otros obstáculos, pero se tiene la convicción de que se trata de una auténtica cuestión de supervivencia. Esta poderosa razón llevó a la creación de los Estados Unidos de América y podría conducir a unos Estados Unidos de Europa, en los parámetros que sintéticamente se han precisado.

Es probable que, como señalaba el profesor Rubio Llorente en su discurso de investidura como doctor honoris causa por la Universidad de Valladolid, el Derecho Constitucional encuentre su mayor reto en el embate que la globalización desencadena en el concepto racional normativo de Constitución ${ }^{7}$.

7 RuBio Llorente, F., «Globalización económica y reforma constitucional», Revista de Occidente, núm. 388, 2013, pp. 5-20. Aunque el recientemente fallecido maestro hablará literalmente de «...incidencia que la globalización económica tiene sobre los Esta- 
Alcanzar los objetivos finales de este proyecto de investigación, o de los sucesivos, intenta ser una modesta contribución a la ardua tarea que tiene el Derecho constitucional y que debe sumarse a la de otras disciplinas jurídicas y sociales, algunas de ellas ya presentes en el proyecto. No obstante, para alcanzar esos objetivos es necesario afrontar, previamente, a modo de estudios preliminares, toda una serie de conceptos, instituciones y figuras; muchos de éstos completamente alejados de la tradición constitucional pero que precisan de análisis desde esta perspectiva.

El estudio de estos conceptos e instituciones requiere, en primer lugar, determinar rigurosamente los modelos de constitución económica así como el marco jurídico de la Unión Europea en esta materia, poderosamente influenciado por el ordoliberalismo. En segundo lugar, determinar, estudiar y diferenciar las diversas fases de integración, dilucidando si es necesario distinguir entre una Unidad económica supraestatal y una Unidad económica y monetaria supraestatal. Realizar un prontuario mundial de los diferentes tipos de integración y estudiar los más relevantes. En tercer lugar, elaborar una teoría general de los acuerdos de libre comercio de la segunda etapa de la globalización en la que no sólo cambian los actores sino también los contenidos (órganos de gobierno, intensa implementación legislativa, órganos jurisdiccionales o arbitrales); así como estudiar los acuerdos de libre comercio e inversiones entre la Unión Europea y Estados Unidos y el de Canadá, que se encuentra más avanzado. Estos trabajos preliminares desembocan, en cuarto lugar, en el análisis de las nuevas instituciones de gobierno de la economía mundial, desde una perspectiva también constitucional, lo que exige ocuparse de los bancos centrales, que en la fijación de la paridad internacional de la divisas ocupan un papel central, cada vez más alejado del principio democrático; de la Organización Mundial del Comercio, instrumento por excelencia de la expansión de la globalización; de cómo junto al G 7 aparece el G 20, lo que sólo se explica en clave globalizadora; de la OCDE, del FMI y del Banco Mundial, entre otras instituciones.

Estos estudios preliminares deben permitir un enfoque diferente de cuatro problemas centrales, que a su vez están profundamente interrelacionados entre sí. El marco económico de la Unión europea en clave ordoliberal y la desconstitucionalización asimétrica y progresiva de las constituciones económicas intervencionistas europeas de los Estados miembros, lo que origina un peligroso estrechamiento del pluralismo político en este ámbito material. El segundo problema es consecuencia del anterior, la adopción constitucional de la regla de oro, esto es, la estabilidad presupuestaria. El tercero deriva

dos nacionales» (p. 6). Y reveladoramente precisa: «Sobre todos ellos, aunque no sobre todos con la misma intensidad, ni es igual la disposición de los distintos Estados para afrontarlos, ni la capacidad que tienen para hacerlo con esperanza de éxito» (pp. 6 y 7). 
de ésta y del primer problema, esto es, de la concepción ordoliberal del marco económico (quizás también de la propia globalización) y del rol central de su principio de competitividad, que no sólo juega ad intra de la integración sino ad extra; se está aquí haciendo referencia a emplear como elemento de mejora de la competitividad a la materia tributaria, y, lo que nos sitúa ya en el cuarto problema, a los valores constitucionales, derechos sociales, derechos de los trabajadores y a la protección del medio ambiente entre otros factores; lo que probablemente explica las políticas de austeridad impulsadas por el líder de la agrupación europea para ganar competitividad ad extra, esto es, en el contexto de la segunda etapa de la globalización.

Ésta es la ingente tarea que afronta esta investigación, quizá ahora pueda comprenderse mejor la integración de los proyectos coordinados de Deusto y de la Pablo de Olavide de Sevilla. Es prácticamente seguro que este trabajo no se agote en un único proyecto de investigación; no obstante, sólo el proyecto coordinador de Castilla-La Mancha tiene previsto la celebración de unas jornadas internacionales con cuatro seminarios el próximo mes de junio, otras el 21 y 22 de noviembre, y, finalmente, un Congreso internacional en mayo del próximo año; su actividad, así como la de los proyectos coordinados, puede seguirse en la página web $<w w w . m a r c o 3 . o r g ~>$.

En el generoso y prestigioso marco de la revista Estudios de Deusto se publican a continuación algunos de estos trabajos preparatorios de los cuatro problemas centrales y de los objetivos finales del proyecto de investigación. Siguen el orden de estas líneas introductorias en forma de Estudios y Notas. Son los primeros resultados junto con otros que en forma de ponencia se ha defendido en diversos congresos nacionales e internacionales y que se encuentran en prensa y que podrán seguir en la citada página web.

TITLE: Constitution and markets within the crisis of the European Union: preliminary considerations

RESUMEN: En el nuevo orden mundial globalizado, la competencia entre bloques económicos condiciona la orientación e intensidad del proceso de integración europea afectando a los fundamentos constitucionales de los Estados nación (principio democrático, dignidad humana y Estado social). Nos preguntamos si la solución a estos problemas se encuentra en una mera reforma de los mecanismos de integración o si por el contrario resulta inevitable desembocar en una Europa Federal.

PALABRAS CLAVE: Globalización, integración supranacional, Constitución económica.

ABSTRACT: The competence among economic blocks in the new globalized order affects the direction and intensity of the European integration process and the constitucional foundations of the member States (democratic 
principle, human dignity and welfare state). We ask if the solution to these problems is a mere reform of the integration mechanisms or if, on the contrary, it is inevitable a Federal Europe.

KEY WORDS: Globalization, Supranational Integration, Economic Constitution.

RECIBIDO: 03.06.2016

ACEPTADO: 22.06.2016 